\title{
Efeitos do Treinamento Auditivo em Idosos com Comprometimento Cognitivo Leve
}

\section{Effects of Auditory Training in Elderly with Mild Cognitive Impairment}

\author{
Renata Rezende de Almeida Ávila, Cristina Ferraz Borges Murphy \& Eliane Schochat* \\ Universidade de São Paulo, São Paulo, São Paulo, Brasil
}

\begin{abstract}
Resumo
Introdução: Pesquisas demonstram a existência do Transtorno do Processamento Auditivo em indivíduos com Comprometimento Cognitivo Leve. Objetivo: Verificar os efeitos de um treinamento auditivo, em indivíduos com comprometimento cognitivo leve. Métodos: Participaram 25 indivíduos, com idades entre 69 e 91 anos e diagnóstico de Comprometimento Cognitivo Leve, sendo que, 10 receberam treinamento auditivo (grupo estudo), 10 receberam treinamento visual (grupo alternativo) e cinco não receberam intervenção (grupo controle). Testes cognitivos e de processamento auditivo foram aplicados antes e após os treinamentos. Resultados: Apenas o grupo estudo apresentou melhora significante para todas as habilidades auditivas testadas, mas com piora do desempenho para as habilidades cognitivas. Conclusão: $\mathrm{O}$ treinamento auditivo foi efetivo em relação às habilidades auditivas, mas não em relação às habilidades cognitivas.

Palavras-chave: Reabilitação, percepção auditiva, cognição, demência.
\end{abstract}

\begin{abstract}
Introduction: Studies have shown the presence of an Auditory Processing Disorder in individuals with mild cognitive impairment. Objective: To investigate the effect of an auditory training in individuals with mild cognitive impairment. Methods: Twenty-five individuals with mild cognitive impairment and ages between 69 and 91 participated in the study. They were divided into three groups: a study group (10 subjects) who underwent an auditory training; an alternative group (10 subjects) who underwent to visual training and a control group ( 5 subjects) who did not receive any intervention. Results: After training, only the study group demonstrated better performance regarding all auditory abilities, but worse performance regarding cognitive abilities. Conclusion: Auditory training was effective regarding auditory abilities but not for cognitive abilities.

Keywords: Rehabilitation, auditory perception, cognition, dementia.
\end{abstract}

Segundo Ritchie, Sylvaine e Touchon (2001), déficits cognitivos sem demência têm sido frequentemente considerados como consequência natural do envelhecimento cerebral, apontando para um envelhecimento normal. Porém, alguns indivíduos podem apresentar um comprometimento cognitivo mais evidenciado, mas sem magnitude suficiente para classificá-los como portadores de demência. Assim, esse grupo constitui um estágio intermediário entre o envelhecimento normal e a demência, caracterizando um comprometimento cognitivo leve (CCL).

Os estudos que investigaram as características deste grupo apontam déficits relacionados à memória episódica (Belanger, Belleville, \& Gauthier, 2010), declínio da fluência verbal fonêmica (Doi et al., 2013), da atenção e do controle executivo (Reinvang, Grambaite, \& Espeseth,

\footnotetext{
"Endereço para correspondência: Departamento de Fisioterapia Fonoaudiologia e Terapia Ocupacional, Faculdade de Medicina, Universidade de São Paulo, Rua Cipotânea, 51, Cidade Universitária, São Paulo, SP, Brasil 05360160. E-mail: eschocha@usp.br
}

2012). Além disso, estudos recentes também demonstraram a presença de um Transtorno do Processamento Auditivo, mesmo quando não há perda auditiva associada (Idrizbegovic et al., 2011; Lopes, 2011, Rahman, Mohamed, Albanouby, \& Tomader, 2011).

Idrizbegovic et al., 2011, por exemplo, aplicou testes de processamento auditivo em 55 indivíduos portadores de comprometimento cognitivo leve e comparou a um grupo de indivíduos com Alzheimer e um grupo com queixas envolvendo memória. Não houve diferença significante inter-grupos em relação ao desempenho na audiometria tonal e testes de percepção de fala no silêncio e no ruído. Entretanto, em relação ao teste dicótico de dígitos, houve diferença significante inter-grupos com pior desempenho para grupo com Alzheimer, seguido de grupo com CCL e por fim, o grupo com queixas de memória. Os autores concluíram que o Transtorno de Processamento Auditivo já é aparente em grupo com CCL e que o teste dicótico de dígitos serviu como um indicador na identificação precoce do grupo de risco para a doença de Alzheimer ou para o comprometimento cognitivo leve. Rahman et al. (2011) 
também aplicou testes de processamento auditivo em 150 indivíduos com CCL e comparou a um grupo controle. O grupo com CCL apresentou pior desempenho, com diferença significante, em relação aos testes de atenção auditiva seletiva, dicótico de dígitos (orelha esquerda), padrão de frequência e memória auditiva demonstrando um declínio do desempenho relacionado às habilidades auditivas.

Em termos de reabilitação do CCL, treinos cognitivos são propostos como método terapêutico e tem se mostrado efetivos (Belleville et al., 2011; Buschert et al., 2011; Förster et al., 2011; Hampstead, Stringer, Stilla, Giddens, \& Sathian, 2012; Herrera, Chambon, Michel, Paban, \& Alescio-Lautier, 2012; Rosen, Sugiura, Kramer, Whitfield-Gabrieli, \& Gabrieli, 2011). Já em relação ao transtorno do processamento auditivo, não há estudos visando à reabilitação destas habilidades. Apesar disso, esta intervenção é fundamental se considerarmos o impacto do transtorno do processamento auditivo nas habilidades de linguagem e na comunicação em geral (Idrizbegovic et al., 2011; Rahman et al., 2011). Além disso, há uma série de hipóteses que relacionam os aspectos auditivos e cognitivos. Discute-se, por exemplo, se as perdas auditivas e cognitivas coexistem em função de um mesmo processo degenerativo, se o declínio cognitivo conduz aos prejuízos de percepção auditiva, ou se a diminuição da percepção auditiva compromete transitoriamente o desempenho cognitivo (Baltes \& Lindenberger, 1997). Assim, considerando as hipóteses descritas, também poderíamos supor que, talvez, o treinamento auditivo também possa influenciar positivamente as habilidades cognitivas.

O objetivo da presente pesquisa é investigar o efeito de um treinamento auditivo nas habilidades auditivas e cognitivas, em indivíduos com CCL. Além disso, também será investigada a existência de correlações, antes e após o treino, entre os aspectos auditivos e cognitivos.

\section{Método}

\section{Participantes}

Participaram do estudo 25 indivíduos, de ambos os sexos, com idades entre 69 e 91 anos e diagnóstico de CCL.

Os indivíduos foram recrutados por meio do Programa Reouvir (Serviço de Otorrinolaringologia) e no Ambulatório de Memória do Idoso (Serviço de Geriatria) e deveriam apresentar idade igual ou superior a 60 anos e diagnóstico de comprometimento cognitivo leve de acordo com a Escala de Avaliação Clínica de Demência (CDR). Além disso, como critério de exclusão, não deveriam apresentar sinais clínicos de demência e pontuação no Miniexame do Estado Mental (MEEM; Folstein, Folstein, \& McHugh, 1975) inferior àquela esperada para a escolaridade, qualquer doença neurológica ou psiquiátrica, bem como causas secundárias de demência, uso de medicamentos com ação no sistema nervoso central, uso de inibidores da acetilcolinesterase, uso de prótese auditiva, média dos limiares audiológicos superiores a $50 \mathrm{~dB}$ nas frequências de 500, 1000, 2000, 3000 e $4000 \mathrm{~Hz}$ e acuidade visual prejudicada a ponto de interferir na realização dos testes de processamento auditivo que continham estímulos visuais.

\section{Procedimentos}

Anteriormente à coleta de dados, o estudo foi aprovado pela Comissão de Ética para Análise de Projetos de Pesquisa (CAPPesq) do Hospital das Clínicas e da Faculdade de Medicina da Universidade de São Paulo (FMUSP), sob o protocolo de pesquisa $n^{\circ} 720 / 06$.

Primeiramente, foi realizada uma consulta aos prontuários dos indivíduos do ambulatório de geriatria, considerando os critérios já descritos. Um dos critérios foi diagnóstico de comprometimento cognitivo leve de acordo com a Escala de Avaliação Clínica de Demência (CDR). Esta escala avalia a cognição e comportamento, além da influência das perdas cognitivas na capacidade de realizar adequadamente as atividades da vida diária. Esse instrumento está dividido em seis categorias funcionais: memória, orientação, juízo e resolução de problemas, assuntos comunitários, atividades domésticas e hobbies e cuidado pessoal. A partir do desempenho, é possível avaliar o estadiamento do estado evolutivo da demência, apontando em uma variação de 0 a 3 - (0) saudável, $(0,5)$ questionável, (1) leve, (2) moderado, (3) grave - o grau de comprometimento funcional causado pelo acometimento em questão (Morris, 1993). Para o diagnóstico do CCL, os indivíduos deveriam apresentar pontuação de 0,5 , caracterizando estado evolutivo de demência questionável. Os indivíduos convocados foram submetidos, em duas sessões, a uma série de procedimentos: anamnese, Avaliação Cognitiva (CDR, MEEM, CAMCOG, TCMR e Dígitos), Avaliação Audiológica (audiometria tonal, vocal e imitanciometria) e Avaliação de PA (Fala com Ruído, Dicótico Não-Verbal, SSW, SSI). A partir dos resultados destas avaliações, foram excluídos os indivíduos que não se enquadravam aos critérios descritos.

Após esta seleção, os participantes foram distribuídos visando à formação dos grupos: grupo estudo (que recebeu o treinamento auditivo), grupo controle (que não recebeu nenhum tipo de treinamento) e grupo alternativo (que receberá um treinamento alternativo, no caso, um treinamento visual). A inclusão deste último grupo é importante para que, caso haja uma melhora pós-treino no desempenho do grupo estudo, possamos assegurar que está é decorrente especificamente deste tipo de treino sensorial e não de qualquer tipo de treinamento a ser realizado. Já o grupo controle permitirá investigarmos a presença de um efeito teste-reteste, que poderia ocorrer como decorrência da reaplicação dos mesmos testes após o treino. Nesta distribuição, adotou-se como critério o pareamento dos grupos em relação à idade e os resultados nas avaliações audiológicas, avaliação de PA e avaliações cognitivas. 
Schochat, E. (2014). Efeitos do Treinamento Auditivo em Idosos com Comprometimento Cognitivo Leve.

\section{Instrumentos}

A seguir serão descritos os testes cognitivos e auditivos aplicados antes e após o período de treinamento e utilizados como parâmetros para a análise dos efeitos das intervenções aplicadas.

\section{Avaliação Cognitiva}

Seção Cognitiva do Cambridge Examination for Mental Disorders of the Elderly (CAMCOG) - Teste cognitivo pertencente ao CAMDEX (Cambridge Mental Disorders of Elderly Examination). Avalia orientação, linguagem, memória, praxia, atenção, pensamento abstrato, percepção e cálculo. É composto por 67 itens, cuja pontuação varia de 0 a 107 (Bottino et al., 2001; Roth et al., 1986).

Teste Comportamental de Memória de Rivermead (TCMR). Teste de memória que consiste em uma bateria de tarefas práticas que procura simular situações cotidianas, diminuindo, assim, a interferência de fatores culturais. Contém 12 subtestes, cujos resultados brutos são convertidos em escore de perfil padronizado com o máximo possível de 24 pontos (Wilson, Cockbum, \& Baddley, 1991).

Teste de Amplitude de Dígitos (Wechler Adult Intelligence Scale). Aplicado na forma de dígitos diretos e indiretos que avaliam a capacidade de concentração e a memória de trabalho, respectivamente. A pontuação varia de 0 a 14 considerando ambas as aplicações (Wechsler, 1981). Avaliação de Processamento Auditivo (PA)

Teste Fala com Ruído. Avalia as habilidades de atenção seletiva e fechamento auditivo através de uma lista de 25 monossílabos, apresentada ipsilateralmente, na intensidade de $40 \mathrm{dBNS}$ (a partir do limiar de reconhecimento de fala). O ruído branco foi apresentado, concomitante aos monossílabos, considerando a relação sinal/ruído de $+20 \mathrm{~dB}$ (Pereira \& Schochat, 1997).

Teste Dicótico Não-Verbal (DNV). Avalia a habilidade de atenção seletiva a partir de uma tarefa de escuta dicótica. Foram apresentados, de forma bilateral, diferentes sons ambientais, na mesma intensidade (50dBNS), e os indivíduos foram instruídos a associar o som ouvido em uma determinada orelha, a uma figura correspondente (total de itens $=12$; Pereira \& Schochat, 1997). Anteriormente ao início do exame, todo o material visual utilizado foi apresentado a cada sujeito para investigação se este é capaz de visualizá-lo.

Spondaic Staggered Words (SSW). Avalia a habilidade de figura-fundo e integração binaural. Foram apresentadas 40 sequências de quatro palavras dissílabas paroxítonas, dentre as quais duas são apresentadas em condição competitiva a $50 \mathrm{dBNS}$. Os indivíduos foram instruídos a repetir as quatro palavras na mesma sequência em que foram apresentadas (Pereira \& Schochat, 1997).

Synthetic Sentence Speech (SSI). Avalia a habilidade de figura-fundo através de tarefa monótica. Os indivíduos foram instruídos a localizar em um quadro a representação gráfica que corresponde a uma sentença ouvida enquanto outra mensagem foi apresentada concomitantemente, na mesma orelha a $40 \mathrm{dBNS}$ (total de itens $=10$; Pereira $\&$ Schochat, 1997).

Logo após as avaliações, os grupos estudo e alternativo foram encaminhados aos programas de intervenção auditivo (grupo estudo) e visual (grupo alternativo).

Ambos foram realizados em oito sessões, uma vez por semana, com duração de 40 minutos cada. Ao fim de cada programa, os grupos foram novamente submetidos às avaliações de PA e Avaliações Cognitivas. Neste mesmo período, o grupo controle não foi submetido a nenhum tipo de intervenção, sendo reavaliado após oito semanas. A seguir são descritos detalhes relacionados a cada um dos treinos realizados:

Treino Auditivo. Este programa de treinamento foi baseado em procedimento proposto por Chermak e Musiek (1992) e Musiek e Chermak (1995), sendo validado posteriormente por Musiek e Schochat (1998). O objetivo deste treino foi reabilitar ou estimular habilidades auditivas como atenção seletiva, memória auditiva, figura-fundo, fechamento auditivo, processamento temporal e integração binaural, por meio de tarefas facilitadas na qual o índice de acerto/erro se mantenha em aproximadamente 70/30\%. Um exemplo de estratégia foi a facilitação da relação sinal/ruído para o treino de habilidades como fechamento auditivo e figura-fundo. Outro exemplo foi o aumento da intensidade na orelha para a qual a escuta deveria ser direcionada, em testes de escuta dicótica, visando o treino da habilidade de atenção seletiva. $\mathrm{O}$ treino foi realizado em cabine acústica através de uma ampla gama de materiais (compact discs) e sempre considerando tarefas diferentes das que foram utilizadas durante as avaliações.

Treino Visual. Este treino foi realizado de forma análoga ao treino auditivo já que as mesmas habilidades foram consideradas durante as sessões como fechamento visual, figura-fundo e memória visual, além das habilidades de discriminação de formas e tamanhos. Para a habilidade de fechamento auditivo, um exemplo de tarefas foi completar parte de um desenho ou de uma palavra; para a habilidade de discriminação, os indivíduos deveriam encontrar as representações que mais se assemelhavam entre si; para a habilidade de figura-fundo foram consideradas tarefas de caça-palavras.

\section{Análise dos Dados}

O método estatístico utilizado visou à comparação dos três grupos em relação às variáveis: idade, gênero, orelha, grupo e situação (pré e pós-treinamento). Utilizou-se o teste de Kruskal-Wallis para a comparação entre os três grupos estudados; para as variáveis "gênero" e "faixa etária" utilizou-se o teste do qui-quadrado; para comparação entre os desempenhos nos testes auditivos e cognitivos utilizou-se o teste de Mann-Whitney, ajustado pela correção de Bonferroni. Além disso, também foi aplicada a análise de correlação de Spearman para o estudo do relacionamento entre as variáveis auditivas e cognitivas. Adotou-se o nível de significância de 5\% $(0,05)$. 


\section{Resultados}

A Tabela 1 mostra o perfil dos grupos quanto às variáveis idade, audição periférica, PA e cognição. Quanto ao desempenho nos testes auditivos e cognitivos, apenas um teste, em uma das orelhas (Fala com Ruído na orelha direta) apresentou diferença significante entre os grupos $(p=0,023)$.

A Tabela 2 mostra os desempenhos dos três grupos nas Avaliações Cognitivas e de PA aplicadas antes e após o período de treino.

Tabela 1

Perfil dos Grupos quanto às Variáveis Gênero, Idade, Audição Periférica, PAC e Cognição

\begin{tabular}{|c|c|c|c|c|}
\hline Variáveis & $\operatorname{GE}(n=10)$ & GA $(n=10)$ & $\mathrm{GC}(n=5)$ & $p$ \\
\hline Gênero $(n)$ & & & & $0,707^{*}$ \\
\hline Feminino & 7 & 8 & 3 & \\
\hline Masculino & 3 & 2 & 2 & \\
\hline Idade & 82,1 & 73,6 & 76,2 & $0,132^{*}$ \\
\hline \multicolumn{5}{|l|}{ Audição Periférica $(M \pm D P)$} \\
\hline OD & $24,6 \pm 14,3$ & $22,0 \pm 10,0$ & $25,8 \pm 15,8$ & $0,943 * *$ \\
\hline $\mathrm{OE}$ & $24,5 \pm 14,9$ & $20,80 \pm 10,2$ & $24,6 \pm 16,5$ & $0,859^{* *}$ \\
\hline \multicolumn{5}{|l|}{ Avaliação Cognitiva $(M \pm D P)$} \\
\hline CAMCOG $($ total = 105) & $77,7 \pm 6,2$ & $85,0 \pm 7,4$ & $77,0 \pm 6,9$ & $0,065^{* *}$ \\
\hline TCMR (total = 24) & $20,0 \pm 3,2$ & $20,3 \pm 2,5$ & $19,0 \pm 2,1$ & $0,587^{* *}$ \\
\hline Dígitos (total = 14) & $9,1 \pm 3,0$ & $10,0 \pm 1,6$ & $9,8 \pm 2,5$ & $0,271 * *$ \\
\hline \multicolumn{5}{|l|}{ Avaliação de PAC $(M \pm D P)$} \\
\hline \multicolumn{5}{|l|}{ Fala com Ruído (\%) } \\
\hline OD & $69,2 \pm 12,0$ & $78,0 \pm 19,3$ & $56,0 \pm 35,1$ & $0,023 * *$ \\
\hline $\mathrm{OE}$ & $72,8 \pm 17,3$ & $83,6 \pm 20,2$ & $60,0 \pm 32,7$ & $0,097 * *$ \\
\hline \multicolumn{5}{|l|}{ SSW $(\%)$} \\
\hline OD & $72,2 \pm 22,6$ & $74,7 \pm 25,3$ & $73,7 \pm 18,3$ & $0,886^{* *}$ \\
\hline $\mathrm{OE}$ & $57,5 \pm 30,1$ & $71,2 \pm 15,8$ & $68,0 \pm 19,5$ & $0,631^{* *}$ \\
\hline \multicolumn{5}{|l|}{ DNV $($ total $=12)$} \\
\hline OD & $7,9 \pm 3,0$ & $8,6 \pm 2,6$ & $8,2 \pm 2,0$ & $0,80^{* *}$ \\
\hline $\mathrm{OE}$ & $9,6 \pm 3,0$ & $9,3 \pm 3,0$ & $10,6 \pm 1,1$ & $0,817^{* *}$ \\
\hline \multicolumn{5}{|l|}{ SSI $($ total $=10)$} \\
\hline OD & $39,0 \pm 15,9$ & $51,0 \pm 16,63$ & $38,0 \pm 10,95$ & $0,141^{* *}$ \\
\hline $\mathrm{OE}$ & $46,0 \pm 22,7$ & $55,0 \pm 17,16$ & $48,0 \pm 13,04$ & $0,609 * *$ \\
\hline
\end{tabular}

Nota. Grupo estudo (GE); Grupo Alternativo (GA); Grupo controle (GC); número de participantes (n); Média (M); Desvio Padrão $(D P)$; Orelha direita (OD); Orelha esquerda (OE); Cambridge Cognition Exam (CAMCOG); Teste Comportamental de Memória de Rivermead (TCMR); Processamento Auditivo Central (PAC); Teste Dicótico de Dissílabos Alternados (SSW); Teste Dicótico Não-Verbal (DNV); Teste de Identificação de Sentenças Sintéticas com Mensagem Competitiva (SSI); * $\mathrm{X}^{2}$ (qui-quadrado); **Teste $U$ de Mann-Whitney. 
Schochat, E. (2014). Efeitos do Treinamento Auditivo em Idosos com Comprometimento Cognitivo Leve.

Tabela 2

Desempenhos Pré e Pós-Treino nas Avaliações Cognitivas e de Processamento Auditivo para os Três Grupos Pesquisados

\begin{tabular}{|c|c|c|c|c|c|c|}
\hline & \multicolumn{2}{|c|}{$\operatorname{GE}(n=10)$} & \multicolumn{2}{|c|}{$\mathrm{GA}(n=10)$} & \multicolumn{2}{|c|}{$\mathrm{GC}(n=5)$} \\
\hline & pré & pós & pré & pós & pré & pós \\
\hline \multicolumn{7}{|l|}{ Avaliação Cognitiva $(M \pm D P)$} \\
\hline CAMCOG $($ total $=105)$ & $77,7 \pm 6,2$ & $71,5 \pm 9,8$ & $85,0 \pm 7,4$ & $81,4 \pm 10,0$ & $77,0 \pm 6,9$ & $74,8 \pm 9,0$ \\
\hline$p$ & \multicolumn{2}{|c|}{$0,030^{*}$} & \multicolumn{2}{|c|}{$0,041^{*}$} & \multicolumn{2}{|c|}{$0,500^{*}$} \\
\hline TCMR $($ total $=24)$ & $20,0 \pm 3,2$ & $18,0 \pm 4,2$ & $20,3 \pm 2,5$ & $19,6 \pm 3,6$ & $19,0 \pm 2,1$ & $18,0 \pm 5,3$ \\
\hline$p$ & \multicolumn{2}{|c|}{$0,051^{*}$} & \multicolumn{2}{|c|}{$0,277^{*}$} & \multicolumn{2}{|c|}{$0,715^{*}$} \\
\hline Dígitos (total = 14) & $9,1 \pm 3,0$ & $9,8 \pm 2,8$ & $10,0 \pm 1,6$ & $13,0 \pm 2,8$ & $9,8 \pm 2,5$ & $9,4 \pm 1,5$ \\
\hline$p$ & \multicolumn{2}{|c|}{$0,196^{*}$} & \multicolumn{2}{|c|}{$0,019^{*}$} & \multicolumn{2}{|c|}{$0,414^{*}$} \\
\hline \multicolumn{7}{|l|}{ Avaliação de $\mathrm{PAC}(M \pm D P)$} \\
\hline \multicolumn{7}{|l|}{ Fala com Ruído (\%) } \\
\hline OD & $69,2 \pm 12,0$ & $84,0 \pm 7,06$ & $78,0 \pm 19,3$ & $63,6 \pm 21,7$ & $56,0 \pm 35,1$ & $38,4 \pm 28,0$ \\
\hline$p$ & \multicolumn{2}{|c|}{$0,005^{*}$} & \multicolumn{2}{|c|}{$0,005^{*}$} & \multicolumn{2}{|c|}{$0,042 *$} \\
\hline $\mathrm{OE}$ & $72,8 \pm 17,3$ & $87,2 \pm 7,5$ & $83,6 \pm 20,2$ & $68,0 \pm 19,0$ & $60,0 \pm 32,7$ & $52,0 \pm 19,3$ \\
\hline$p$ & \multicolumn{2}{|c|}{$0,012 *$} & \multicolumn{2}{|c|}{$0,008^{*}$} & \multicolumn{2}{|c|}{$0,225^{*}$} \\
\hline \multicolumn{7}{|l|}{ SSW (\%) } \\
\hline OD & $72,2 \pm 22,6$ & $86,2 \pm 12,5$ & $74,7 \pm 25,3$ & $81,2 \pm 22,4$ & $73,7 \pm 18,3$ & $67,5 \pm 20,9$ \\
\hline$p$ & \multicolumn{2}{|c|}{$0,021^{*}$} & \multicolumn{2}{|c|}{$0,151^{*}$} & \multicolumn{2}{|c|}{$0,131^{*}$} \\
\hline $\mathrm{OE}$ & $57,5 \pm 30,1$ & $78,5 \pm 16,0$ & $71,2 \pm 15,8$ & $74,6 \pm 16,3$ & $68,0 \pm 19,5$ & $68,5 \pm 18,5$ \\
\hline$p$ & \multicolumn{2}{|c|}{$0,005^{*}$} & \multicolumn{2}{|c|}{$0,312^{*}$} & \multicolumn{2}{|c|}{$0,891 *$} \\
\hline \multicolumn{7}{|l|}{$\mathrm{DNV}($ total $=12)$} \\
\hline OD & $7,9 \pm 3,0$ & $11,1 \pm 0,9$ & $8,6 \pm 2,6$ & $9,5 \pm 3,2$ & $8,2 \pm 2,0$ & $8,2 \pm 2,0$ \\
\hline$p$ & \multicolumn{2}{|c|}{$0,011^{*}$} & \multicolumn{2}{|c|}{$0,156^{*}$} & 0,1 & \\
\hline $\mathrm{OE}$ & $9,6 \pm 3,0$ & $11,6 \pm 0,9$ & $9,3 \pm 3,0$ & $11,0 \pm 1,6$ & $10,6 \pm 1,1$ & $10,6 \pm 1,1$ \\
\hline$p$ & & & & $16^{*}$ & 0,1 & \\
\hline SSI $($ total $=10)$ & & & & & & \\
\hline OD & $39,0 \pm 15,9$ & $73,0 \pm 14,1$ & $51,0 \pm 16,6$ & $52,0 \pm 18,7$ & $38,0 \pm 10,9$ & $38,0 \pm 10,9$ \\
\hline$p$ & & & $>0$ & $99 *$ & $>0$ & $99 *$ \\
\hline $\mathrm{OE}$ & $46,0 \pm 22,7$ & $73,0 \quad 16,3$ & $55,0 \pm 17,1$ & $52,018,7$ & $48,0 \pm 13,0$ & $48,013,0$ \\
\hline$p$ & & & & $80^{*}$ & 0,1 & \\
\hline
\end{tabular}

Nota: Grupo estudo (GE); Grupo alternativo (GA); Grupo controle (GC); número de participantes (n); Média (M); Desvio Padrão $(D P)$; Orelha direita (OD); Orelha esquerda (OE); Cambridge Cognition Exam (CAMCOG); Teste Comportamental de Memória de Rivermead (TCMR); Processamento Auditivo CentraL (PAC); Teste Dicótico de Dissílabos Alternados (SSW); Teste Dicótico Não-Verbal (DNV); Teste de Identificação de Sentenças Sintéticas com Mensagem Competitiva (SSI); *Teste $U$ de Mann-Whitney. 
Para o grupo estudo (GE), após o treino, houve piora significante para o teste CAMCOG $(p=0,03)$ e piora do desempenho com tendência à significância para o teste TCMR $(p=0,051)$. Já para os testes auditivos, houve melhora significante do desempenho para todas as habilidades.

Para o grupo alternativo (GA), após o treino, houve piora significante para o teste CAMCOG $(p=0,041)$ e melhora significante em relação ao Dígitos $(p=0,019)$. Em relação aos testes auditivos, houve piora significante do desempenho para os testes Fala com Ruído (OD - $p=0,005$ / $\mathrm{OE}-p=0,008)$ e melhora significante do desempenho da orelha direita no Teste DNV (OE $-p=0,016)$.

O grupo controle (GC) apresentou, após o treino, piora significante de desempenho da orelha direita no Teste Fala com Ruído $(p=0,042)$.

Também foi analisada a existência de correlações entre os desempenhos nos testes de PA e nos testes cognitivos antes e após o treino.

Em relação aos desempenhos obtidos anteriormente ao treino, no GE, foram encontradas correlações significantes entre os testes Fala com Ruído (OD) e CAMCOG (coef $=0,864, p=0,001$ ), Fala com Ruído (OD) e TCMR (coef $=0,748, p=0,013)$, DNV (OD) e CAMCOG (coef $=0,652, p=0,041$ ), SSW (OD) e CAMCOG $($ coef $=0,792, p=0,006)$ e SSW (OE) e Dígitos (coef= $-0,681, p=0,030)$. Após o treino, houve correlações significantes entre DNV (OD) e CAMCOG (coef $=0,714$, $p=0,020)$, DNV (OD) e Dígitos (coef. $=0,648, p=0,043$ ), DNV (OE) e CAMCOG (coef $=0,679, p=0,031$ ), SSW(OD) e CAMCOG (coef $=0,874, p=0,001)$, SSI (OD) e CAMCOG (coef $=0,675, p=0,032)$ e SSI (OE) e CAMCOG (coef $=0,817, p=0,004$ ).

Para GA, antes do treino, houve correlações significantes entre os testes Fala com Ruído (OD) e CAMCOG (coef $=0,864, p=0,001$ ), Fala com Ruído (OE) e TCMR (coef $=0,748, p=0,013$ ), SSW (OD) e CAMCOG (coef $=0,792, p=0,006)$ e SSW (OE) e Dígitos (coef= $-0,681, p=0,030$ ). Após o treino, as correlações significantes foram entre DNV (OD) e CAMCOG (coef $=0,714$, $p=0,020)$, DNV (OD) e Dígitos (coef $=0,648, p=0,043$ ), DNV (OE) e CAMCOG (coef $=0,820, p=0,004)$, SSW (OD) e CAMCOG (coef $=0,874, p=0,001)$, SSI (OD) e CAMCOG ( coef $=0,675, p=0,032)$ e SSI (OE; coef $=0,817$, $p=0,004)$.

Para GC, antes do período de treino, houve correlações significantes entre SSW(OD) e Dígitos (coef $=0,975$, $p=0,005)$ e SSW (OE) e Dígitos (coef $=0,900, p=0,037$ ). Após o treino, as correlações significantes foram entre os testes Fala com Ruído (OE) e CAMCOG (coef $=0,900$, $p=0,037$ ) e Fala com Ruído (OE) e TCMR (coef $=-0,975$, $p=0,005$ ).

\section{Discussão}

A presente pesquisa analisou o efeito do treinamento auditivo em grupo de indivíduos com CCL, a partir da comparação entre grupo controle e grupo alternativo.
Para discussão dos resultados, serão considerados primeiramente os dados obtidos no pré-treinamento (perfil dos grupos) e, posteriormente, os achados em relação à comparação pré e pós-treinamento para as habilidades auditivas e cognitivas, bem como a correlação entre os dois aspectos.

\section{Perfil dos Grupos}

Anteriormente ao treino, os três grupos pesquisados apresentaram mesmo perfil, ou seja, ausência de diferenças significantes para as variáveis gênero, sexo e audição periférica, além de também apresentarem desempenhos semelhantes em relação a todos os testes aplicados (auditivos e cognitivos), exceto para Fala com Ruído na orelha direita (Tabela 1). Este dado é importante pois demonstra que todos iniciaram o treinamento apresentando aparentemente o mesmo nível de dificuldade do ponto de vista cognitivo e auditivo. Em relação às habilidades auditivas, todos os grupos apresentaram desempenho abaixo do padrão da normalidade (Pereira \& Schochat, 1997), exceto para o Teste Fala com Ruído. Este achado corrobora estudos anteriores na qual a Alteração de Processamento Auditivo também foi diagnosticada em idosos (Idrizbegovic et al., 2011; Lopes, 2011; Rahman et al., 2011) e, além disso, ressalta a importância do treinamento auditivo nesta população. Quanto às habilidades cognitivas, para o teste CAMCOG, todos os três grupos apresentaram média de acerto inferior à média publicada em estudos anteriores sobre CCL; enquanto que no estudo atual a média esteve entre 77 e $85 \%$, no estudo de Nunes et al. (2008), por exemplo, a média foi de $91 \%$ e no estudo de Lopes (2011) $81 \%$, valores sempre menores do que os obtidos para grupo de indivíduos normais. Já o teste Dígitos apresentou valores semelhantes aos obtidos por Lopes (2011; média entre 9,1 e 10), valores que segundo o mesmo autor são semelhantes aos obtidos em indivíduos normais e o teste TCMR, no estudo atual, apresentou valores superiores aos obtidos por Lopes (2011; aproximadamente 16). Assim, a maioria dos testes cognitivos aplicados demonstrou a presença de declínio cognitivo, confirmando a presença do CCL.

\section{Comparação Pré e Pós-Treinamento para as Habilidades Auditivas e Cognitivas}

Habilidades Auditivas. A partir dos resultados encontrados podemos sugerir que: (a) o treinamento auditivo, além de melhorar o desempenho em relação às habilidades auditivas, dado observado no grupo estudo, pode impedir a progressão do declínio destas habilidades, dado presente para grupos controle e alternativo no teste Fala com Ruído; (b) não houve efeito teste-reteste no grupo controle, demonstrando que, provavelmente, a melhora presente no grupo estudo pode ser atribuída ao treino realizado e não a um efeito aprendizagem decorrente da reavaliação; (c) a ausência de influência do treino visual nas habilidades auditivas demonstrou que a melhora do desempenho do grupo estudo pode ser atribuída especificamente ao treino 
Schochat, E. (2014). Efeitos do Treinamento Auditivo em Idosos com Comprometimento Cognitivo Leve.

auditivo realizado neste grupo e não a qualquer tipo de intervenção.

Segundo Musiek e Berger (1998), a melhora comportamental observada após a realização do treinamento auditivo não está relacionada às modificações nos processos auditivos periféricos e sim ao sistema nervoso auditivo central. Essa modificação seria atribuída à plasticidade neural, que geraria uma alteração das células nervosas para acomodar as influências ambientais imediatas, estando tal alteração frequentemente associada a uma modificação comportamental, como observado nos testes aplicados pós-treino. Especificamente na presente pesquisa, a melhora pós-treino das habilidades auditivas corrobora as afirmações de Calero e Navarro, 2004, que afirmam que indivíduos com comprometimento cognitivo leve ainda apresentam neuroplasticidade suficiente para levar às mudanças decorrentes de treinamentos.

A melhora significante do grupo alternativo, especificamente no Teste Dicótico Não-Verbal, talvez possa ser explicada pela relação entre o treinamento que o grupo recebeu (visual) e às características do teste aplicado, já que este é o único que apresenta figuras, as quais devem ser associados a um som não-verbal específico. Já o Teste Fala com Ruído demonstrou ser o teste mais sensível para a observação do declínio do desempenho das habilidades auditivas, já que este foi o único com desempenho estatisticamente pior no pós-treino tanto para o grupo alternativo quanto para o grupo controle. Neste caso, o declínio desta habilidade auditiva (fechamento auditivo), além de poder estar associado ao declínio cognitivo característico do compromentimento cognitivo leve, também pode estar relacionado ao próprio processo de envelhecimento do sistema nervoso auditivo em idosos, que acarreta mudanças nos limiares relacionados à habilidade de fusão auditiva e na percepção de diferenças binaurais, resultando em perda funcional das habilidades de compreensão de fala em condições de escuta desfavoráveis e localização com precisão da fonte sonora (Babkoff et al., 2002).

Habilidades Cognitivas. A piora do desempenho no grupo estudo para 2 dos 3 testes aplicados demonstrou que, de maneira geral, o treinamento auditivo não foi capaz de influenciar positivamente estas habilidades e nem ao menos impedir o declínio; além disso, também evidenciou a rápida evolução da doença. De acordo com Petersen et al. (2001), o risco de conversão do CCL para a Doença de Alzheimer é de $12 \%$ ao ano. No caso da presente pesquisa, a piora significante foi demonstrada em apenas 9 semanas decorridas entre a primeira e segunda avaliação.

A ausência de melhora das habilidades cognitivas no GE demonstra que não houve generalização do aprendizado ocorrido (e comprovado pela melhora das habilidades auditivas) para as habilidades cognitivas. A hipótese considerada estaria relacionada à influência negativa do comprometimento cognitivo leve neste processo de generalização.
Correlações, Antes e Após o Treino, entre os Aspectos Auditivos e Cognitivos

De acordo com os resultados, houve correlações significantes no pré e pós treino em relação às habilidades cognitivas e auditivas, para todos os grupos pesquisados, sugerindo uma relação entre os dois aspectos pesquisados. Esta relação pode estar relacionada às características em comum entre os testes cognitivos e auditivos aplicados, como por exemplo, a memória auditiva, habilidade que deve apresentar-se necessariamente íntegra para o bom desempenho em ambos os aspectos avaliados. Além disso, esta habilidade também foi estimulada durante o treinamento auditivo, o que nos levaria a pensar que, desta forma, tanto os aspectos auditivos quanto cognitivos seriam influenciados positivamente no pós-treino. Murphy et al. (2011), por exemplo, demonstraram que, após o treinamento auditivo, aplicado em indivíduo com traumatismo crânio-encefálico, houve melhora significante em cinco das seis habilidades auditivas avaliadas e em cinco das sete habilidades cognitivas, demonstrando a presença de generalização do aprendizado sensorial para as habilidades cognitivas. Os autores concluíram, portanto, que mesmo a partir de um treinamento unimodal, que teve como objetivo melhorar a percepção dos sinais acústicos,foi possível observar uma influência positiva em habilidades consideradas "top-down", envolvendo tarefas multimodais.

Assim, considerando os resultados da presente pesquisa, podemos sugerir que, talvez, o fator que interferiu na observação desta generalização foi a presença do comprometimento cognitivo leve. Conforme já citado, indivíduos com comprometimento cognitivo leve ainda podem apresentam neuroplasticidade (Calero \& Navarro, 2004), mas, talvez, não apresentem processos suficientes para que este aprendizado seja generalizado para outras habilidades relacionadas. Isto pode estar relacionado a uma das características do declínio cognitivo, que é uma disfunção no centro executivo da memória operacional, responsável por prejudicar o desenvolvimento de estratégias de codificação de material novo e a busca de informações previamente armazenadas (Bennett et al., 2002; Elias et al., 2000).

Diferentemente de GE, GA apresentou melhora significante para um dos testes aplicados (WAIS). Este resultado pode ser atribuído ao treinamento visual recebido já que, assim como no treinamento auditivo foram estimuladas habilidades relacionadas à memória auditiva; no treinamento visual, esta mesma habilidade também foi considerada para a modalidade visual. A possível explicação para presença da generalização, apenas neste grupo, possa estar relacionada à média de idade do grupo e ao desempenho observado no pré-teste; observa-se que apesar de não haver diferenças significantes entre o perfil dos grupos, conforme já comentado no início da Discussão, grupo alternativo obteve melhor desempenho bruto para todos os testes, além apresentar idade média inferior aos outros. Assim, a hipótese considerada é a de que, talvez, estes fatores tenha sido suficientes para a ocorrência de um 
declínio menos acentuado neste grupo especificamente, o que geraria condições para que o processo de generalização ocorresse.

Já em relação ao GC, a não observância de diferenças nos desempenhos obtidos no período pré e pós treino se deve, provavelmente, ao número reduzido de participantes no grupo, o que pode ter influenciado na análise estatística realizada.

Concluindo, o estudo em questão demonstrou a efetividade do treinamento auditivo para a estimulação das habilidades auditivas em indivíduos com CCL. Mas, não houve generalização do aprendizado para as habilidades cognitivas. Futuras pesquisas são necessárias para melhor investigação dos processos de aprendizagem ocorridos nesta população específica.

\section{Referências}

Babkoff, H., Muchnik, C., Ben-David, N., Furst, M., Even-Zohar, S., \& Hildesheimer, M. (2002). Mapping lateralization of click trains in younger and older populations. Hearing Research, 165(1-2), 117-127.

Baltes, P. B., \& Lindenberger, U. (1997). Emergence of a powerful connection between sensory and cognitive functions across the adult life span: A new window to the study of cognitive aging? Psychology and Aging, 12(1), 12-21.

Belanger, S., Belleville, S., \& Gauthier, S. (2010). Inhibition impairments in Alzheimer's disease, mild cognitive impairment and healthy aging: Effect of congruency proportion in a Stroop task. Neuropsychologia, 48, 581-590.

Belleville, S., Clément, F., Mellah, S., Gilbert, B., Fontaine, F., \& Gauthier, S. (2011). Training-related brain plasticity in subjects at risk of developing Alzheimer's disease. Brain, 134(6), 1623-1634.

Bennett, D. A., Wilson, R. S., Schneider, J. A., Evans, D. A., Beckett, L. A., Aggarwal, N. T., ...Bach, J. (2002). Natural history of mild cognitive impairment in older persons. Neurology, 59(2), 198-205.

Bottino, C. M. C., Stoppe, A., Jr., Scalco, A. Z., Ferreira, R. C. R., Hototian, S. R., \& Scalco, M. Z. (2001). Validade e confiabilidade da versão brasileira do CAMDEX. Arquivos de Neuropsiquiatria, 59(3), 30.

Buschert, V. C., Friese, U., Teipel, S. J., Schneider, P., Merensky, W., Rujescu, D., ...Buerger, K. (2011). Effects of a newly developed cognitive intervention in amnestic mild cognitive impairment and mild Alzheimer's disease: A pilot study. Journal of Alzheimer's Diseases, 25(4), 679-694.

Calero, M. D., \& Navarro, E. (2004). Relationship between plasticity, mild cognitive impairment and cognitive decline. Archives of Clinical Neuropsychology, 19(5), 553-660.

Chermak, G. D., \& Musiek, F. E. (1992). Managing central auditory processing disorders in children and youth. American Journal of Audiology, 1(3), 61-66.

Doi, T., Shimada, H., Makizako, H., Yoshida, D., Shimokata, H., Ito, K., ...Suzuki, T. (2013). Characteristics of cognitive function in early and late stages of amnestic mild cognitive impairment. Geriatrics \& Gerontology Internacional, 13(1), 83-89

Elias, M. F., Beiser, A., Wolf, P. A., Au, R., White, R. F., \& D'Agostino, R. B. (2000). The preclinical phase of Alzheimer disease: A 22-year prospective study of the Framingham Cohort. Archives of Neurology, 57(6), 808-813.
Folstein, M. F., Folstein, S. E., \& McHugh, P. R. (1975). "Minimental state". A practical method for grading the cognitive state of patients for the clinician. Journal of Psychiatric Research, 12(3), 189-198.

Förster, S., Buschert, V. C., Buchholz, H. G., Teipel, S. J., Friese, U., Zach, C., ...Buerger, K. (2011). Effects of a 6-month cognitive intervention program on brain metabolism in amnestic mild cognitive impairment and mild Alzheimer's disease. Journal of Alzheimer's Diseases, 25(4), 695-706.

Idrizbegovic, E., Hederstierna, C., Dahlquist, M., Kämpfe Nordström, C., Jelic, V., \& Rosenhall, U. (2011). Central auditory function in early Alzheimer's disease and in mild cognitive impairment. Age Ageing, 40(2), 249-254.

Hampstead, B. M., Stringer, A. Y., Stilla, R. F., Giddens, M., \& Sathian, K. (2012). Mnemonic strategy training partially restores hippocampal activity in patients with mild cognitive impairment. Hippocampus, 22(8), 1652-1658. doi:10.1002/ hipo. 22006

Herrera, C., Chambon, C., Michel, B. F., Paban, V., \& Alescio-Lautier, B. (2012). Positive effects of computer-based cognitive training in adults with mild cognitive impairment. Neuropsychologia, 50(8), 1871-1881.

Lopes, L. C. (2011). Análise do processamento auditivo central em idosos portadores do comprometimento cognitivo leve (Tese de doutorado, Universidade de São Paulo, SP, Brasil).

Morris, J. C. (1993). The clinical dementia rating (CDR): Current version and scoring rules. Neurology, 43(11), 2412-2414.

Murphy, C. F. B., Fillippini, R., Palma, D., Zalcman, T. E., Lima, J. P., \& Schochat, E. (2011). Auditory training and cognitive functioning in adult with traumatic brain injury. Clinics (São Paulo), 66(4), 713-715.

Musiek, F. E., \& Berge, B. E. (1998). A neuroscience view of auditory training/stimulation and central auditory processing disorders. In M. G. Masters, N. A. Stecker, \& J. Katz (Eds.), Central auditory processing disorders: Mostly management (Vol. 1, Chap. 2, pp. 15-24). Boston, MA: Allyn and Bacon.

Musiek, F. E., \& Chermak, G. D. (1995). Three commonly asked question about central auditory processing disorders. American Journal of Audiology, 4, 15-18.

Musiek, F. E., \& Schochat, E. (1998). Auditory training and central auditory processing disorders. Seminars in Hearing, 19(4), 357-366.

Nunes, P. V., Diniz, B. S., Radanovic, M., Abreu, I. D., Borelli, D. T., Yassuda, M. S., \& Forlenza, O. V. (2008). CAMcog as a screnning tool for diagnosis of mild cognitive impairment and dementia in a Brazilian clinical sample of moderate to high education. International Journal of Geriatric Psychiatry, 23(11), 1127-1133.

Pereira, L. D., \& Schochat, E. (1997). Processamento auditivo central: Manual de avaliação. São Paulo, SP: Lovise.

Petersen, R. C., Doody, R., Kurz, A., Mohs, R. C., Morris, J. C., Rabins, P. V., ...Winblad, B. (2001). Current concepts in mild cognitive impairment. Archives of Neurology, 58(12), 1985-1992.

Rahman, T. T., Mohamed, S. T., Albanouby, M. H., \& Bekhet, H. F. (2011). Central auditory processing in elderly with mild cognitive impairment. Geriatrics \& Gerontology Internacional, 11(3), 304-308.

Reinvang, I., Grambaite, R., \& Espeseth, T. (2012). Executive dysfunction in MCI: Subtype or early symptom. International Journal of Alzheimer's Diseases, 2012, 936272.

Ritchie, K., Sylvaine, A., \& Touchon, J. (2001). Classification criteria for mild cognitive impairment: A population-based validation study. Neurology, 56, 37-42. 
Schochat, E. (2014). Efeitos do Treinamento Auditivo em Idosos com Comprometimento Cognitivo Leve.

Rosen, A. C., Sugiura, L., Kramer, J. H., Whitfield-Gabrieli, S., \& Gabrieli, J. D. (2011). Cognitive training changes hippocampal function in mild cognitive impairment: A pilot study. Journal of Alzheimer's Diseases, 26(3), 349-357.

Roth, M., Tym, E., Mountjoy, C. Q., Huppert, F. A., Hendrie, H., Verma, S., \& Goddard, R. (1986). CAMDEX: Standardized instrument for diagnosis of mental disorders in elderly with special reference to the early detection of dementia. British Journal of Psychiatric, 149, 698-709.

Wechsler, D. (1981). WAIS-R Manual. New York: Psychological Corporation.

Wilson, B. A., Cockburn, J., \& Baddeley, A. (1991). The Rivermead Behavioral Memory Test. Titchfield, UK: Thames Valley Test. 